\title{
Migration of mitochondrial DNA in the nuclear genome of colorectal adenocarcinoma
}

Vinodh Srinivasainagendra ${ }^{1}$, Michael W. Sandel ${ }^{1,7+}$, Bhupendra Singh ${ }^{2 \dagger}$, Aishwarya Sundaresan ${ }^{1}$, Ved P. Mooga ${ }^{2}$, Prachi Bajpai ${ }^{2}$, Hemant K. Tiwari ${ }^{*}$ and Keshav K. Singh ${ }^{3,4,5,6^{*}}$

\begin{abstract}
Background: Colorectal adenocarcinomas are characterized by abnormal mitochondrial DNA (mtDNA) copy number and genomic instability, but a molecular interaction between mitochondrial and nuclear genome remains unknown. Here we report the discovery of increased copies of nuclear mtDNA (NUMT) in colorectal adenocarcinomas, which supports link between mtDNA and genomic instability in the nucleus. We name this phenomenon of nuclear occurrence of mitochondrial component as numtogenesis. We provide a description of NUMT abundance and distribution in tumor versus matched blood-derived normal genomes.
\end{abstract}

Methods: Whole-genome sequence data were obtained for colon adenocarcinoma and rectum adenocarcinoma patients participating in The Cancer Genome Atlas, via the Cancer Genomics Hub, using the GeneTorrent file acquisition tool. Data were analyzed to determine NUMT proportion and distribution on a genome-wide scale. A NUMT suppressor gene was identified by comparing numtogenesis in other organisms.

Results: Our study reveals that colorectal adenocarcinoma genomes, on average, contains up to 4.2-fold more somatic NUMTs than matched normal genomes. Women colorectal tumors contained more NUMT than men. NUMT abundance in tumor predicted parallel abundance in blood. NUMT abundance positively correlated with GC content and gene density. Increased numtogenesis was observed with higher mortality. We identified YME1L1, a human homolog of yeast YME1 (yeast mitochondrial DNA escape 1) to be frequently mutated in colorectal tumors. YME1L1 was also mutated in tumors derived from other tissues. We show that inactivation of YME1L1 results in increased transfer of mtDNA in the nuclear genome.

Conclusions: Our study demonstrates increased somatic transfer of mtDNA in colorectal tumors. Our study also reveals sex-based differences in frequency of NUMT occurrence and that NUMT in blood reflects NUMT in tumors, suggesting NUMT may be used as a biomarker for tumorigenesis. We identify YME1L1 as the first NUMT suppressor gene in human and demonstrate that inactivation of YME1L1 induces migration of mIDNA to the nuclear genome. Our study reveals that numtogenesis plays an important role in the development of cancer.

Keywords: Cancer, Tumor, Colorectal cancer, Mitochondria, Mitochondrial DNA, YME1L1, NUMT, Numtogenesis, mtDNA transfer, Genetic instability

\footnotetext{
*Correspondence: htiwari@uab.edu; kksingh@uab.edu

${ }^{\dagger}$ Equal contributors

${ }^{1}$ Department of Biostatistics, School of Public Health, University of Alabama

at Birmingham, Birmingham, Alabama 35294, USA

${ }^{3}$ Departments of Genetics, Environmental Health, Center for Free Radical

Biology, Center for Aging and UAB Comprehensive Cancer Center, University

of Alabama at Birmingham, Birmingham, Alabama 35294, USA

Full list of author information is available at the end of the article
}

(c) The Author(s). 2017 Open Access This article is distributed under the terms of the Creative Commons Attribution 4.0 International License (http://creativecommons.org/licenses/by/4.0/), which permits unrestricted use, distribution, and reproduction in any medium, provided you give appropriate credit to the original author(s) and the source, provide a link to the Creative Commons license, and indicate if changes were made. The Creative Commons Public Domain Dedication waiver (http://creativecommons.org/publicdomain/zero/1.0/) applies to the data made available in this article, unless otherwise stated. 


\section{Background}

Natural transfer of mitochondrial DNA (mtDNA) into the nuclear genomes of eukaryotic cells is a well-established and evolutionarily ongoing process. The nuclear copies of mtDNA are described as NUMTs (nuclear mtDNA sequences) [1]. Frequently, intact mitochondria containing mtDNA, mitochondrial RNA (mtRNA), and mitochondrial proteins are also reported to localize into the nucleus [2-9]. We have named this phenomenon of occurrence of nuclear mitochondria as numtogenesis. We define numtogenesis as the occurrence of any mitochondrial components into the nucleus or nuclear genome. Numtogenesis is reported in at least 85 sequenced eukaryotic genomes [1]. These include human, plant, yeast, fruit fly, Plasmodium, Caenorhabditis, and other species [1, 10, 11]. In human, NUMT insertions are estimated to occur at a rate of $\sim 5 \times 10^{-6}$ per germ cell per generation [12].

Evolutionary studies suggest that the origin and insertion of germline NUMTs are distributed non-randomly in humans and other mammals [13-15]. Germline NUMTs tend not to originate from the mtDNA displacement loop ("d-loop"), and they tend to be located in damage-prone regions of the nuclear genome, such as open chromatin and fragile sites [13-15]. These studies implicate NUMTs in double-strand break repair [13]. The mechanism(s) of NUMT accumulation is not well understood. It is suggested that mitochondria migrate towards the nucleus and accumulate near the nuclear membrane [16-18]. The most parsimonious mechanism explaining NUMT accumulation involves de novo transposition from the mitochondrion to the nucleus; however, NUMTs are also known to accumulate via segmental duplication (sometimes within repetitive elements), and possibly RNA retro-transposition [19-21]. The human genome contains between 755 and 1105 germline NUMTs, with mtDNA identities ranging from $64-100 \%[12,22]$. Germline NUMTs with the lowest similarity to mtDNA have been evolutionarily conserved for tens of millions of years, while the most recent insertions occurred after certain Homo sapiens populations migrated to Eurasia [22-26]. Human germline NUMTs are relatively well described but little is known about the somatic NUMT and its role in human pathology [27].

We and others have demonstrated that mito-nuclear interactions play a key role in tumorigenesis [28-35], but a role of mtDNA integration within the nuclear genome remains relatively unexplored. In this study, we analyzed the prevalence of NUMT in colorectal cancer (CRC) because a number of mitochondrial associations are relatively well characterized in CRC. There is a reported relationship between CRC risk and mtDNA copy number [36-38], and there are associations between germline mtDNA variants and CRC risk and mortality $[39,40]$. Similarly, colorectal adenocarcinomas tend to have aberrant mtDNA copy number and somatic variant frequencies compared to matched blood-derived normal genomes [41-44]. We differentiated two classes of NUMTs with distinct characteristics, those that are inherited in the germline and those that are somatic NUMTs acquired during tumorigenesis. We present the first quantitative analysis on the abundance of somatic NUMTs in human colorectal adenocarcinoma genomes relative to matched blood-derived normal samples. Further, we compare the distributions of somatic NUMTs and germline NUMTs, describe sex-based differences in numtogenesis, and demonstrate that NUMT abundance in blood reflects NUMT abundance in tumor. In addition, we identify YME1L1 as the first "NUMT suppressor" gene in humans whose inactivation leads to increased numtogenesis.

\section{Methods}

\section{Data harvesting}

Whole-genome sequence data were obtained for colon adenocarcinoma (COAD) and rectum adenocarcinoma (READ) patients participating in The Cancer Genome Atlas (TCGA), via the Cancer Genomics Hub (CGHub) [45], using the GeneTorrent file acquisition tool. In order to appraise alternative protocols for transactions on big-data endpoints, we evaluated GTFuse, an innovate software that offers faster access to DNA sequence data without the need for staging the data locally. Although data bandwidth hungry, GTFuse still enabled us to rapidly prototype our research on selected regions of the genome, thereby indicating signals of NUMT deposition in the nuclear genome. A symbiotic combination of GeneTorrent and GTFuse was used to choreograph the downstream data analysis pipeline. Our data harvester robots adopt a high-throughput analysis model by spreading GTFuse across a computational cluster fabric available locally on-campus At the time of manuscript generation, although the GTFuse web-link within AnnaiSystems webportal was unavailable, an alternate web URL https://annaisystems.zendesk.com/hc/ en-us was found through web search means.

\section{Quality control}

TCGA sequence data for individuals with matched colorectal tumor and blood-derived normal samples were harvested from CGHub. Downloaded data went through an intense quality control (QC) pipeline, which involved the use of (a) clinical information downloaded from TCGA Data Matrix, (b) short-reads alignment statistics derived from the Binary AlignMent (BAM) sequence data downloaded from CGHub, and (c) tagging and elimination of duplicate reads in the alignment data (BAM) using a popular next-generation sequencing tool, Picard 2.5. All sequence data used in this study were generated at Harvard Medical School using Illumina 
paired-end read technologies GAII and HiSeq, and reads were mapped to the hg18 human reference assembly. Since paired-end read technology offers better mapping coverage, improved directional sequence accuracy, and reliable mapping of reads to a reference genome, we used sequencing datasets generated using Illumina's paired-end read technology as opposed to single-end read technology. Paired-end reading, with its increased coverage across several genomic bases, also improves the ability to identify relative positions of the two ends of a single read, especially when each read-end maps to different genomes, nuclear and mitochondrial.

Further, a second-level QC was conducted based on the clinical annotations of the samples (Fig. 1). Participants were excluded a priori if even one of the following conditions as unmet (a) one or more elements of TCGA barcode differed between matched tumor and bloodderived healthy samples (i.e., sample, vial, analyte, and plate identifiers), (b) multiple alignment data (BAM files) per aliquot were reported when using CGHub's sequence data query tool, cgquery, and (c) self-reported race and ethnicity status was not white or black. After the

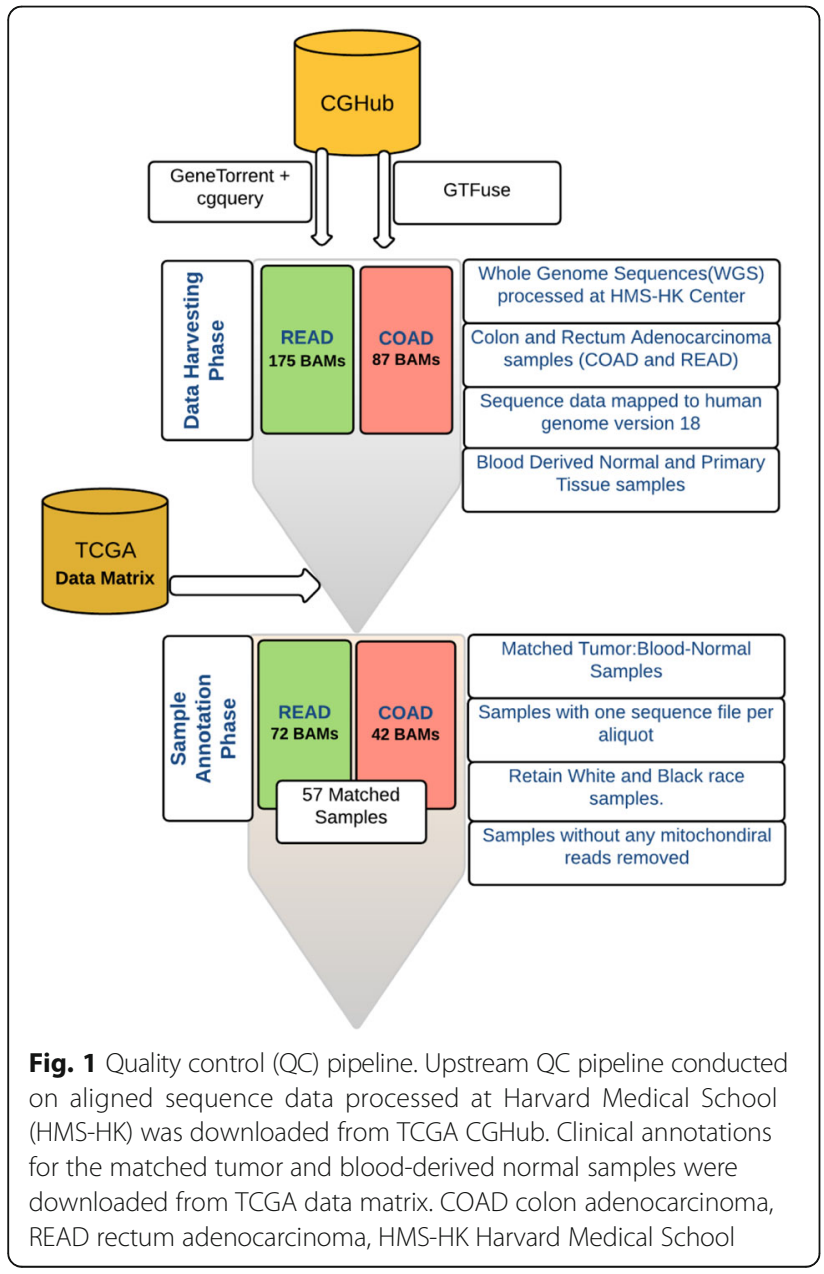

complete QC process, the final dataset included 57 colorectal tumor and 57 matched blood-derived normal genome alignment files (BAM files). Figure 1 illustrates the complete QC pipeline.

\section{Data analytics}

A two-pronged analytic approach is taken to determine (a) NUMT proportion and distribution on a genome-wide scale and (b) hot spots in the nuclear and mitochondrial genomes experiencing more than blood-derived healthy (normal) NUMT abundance.

The primary obstacle to quantifying non-homologous recombinant elements from high-throughput sequence data is the reliable detection of reads that map to the sequence breakpoint. Although conceptually intuitive, the mapping and alignment of breakpoint reads is complicated by the large number of NUMT inserts that are not represented in the reference genome sequence. Alternatively, de novo genome assembly is computationally intensive, and any low-stringency mapping algorithm that would accurately identify insertion sites would also return many false positive hits. Fortunately, paired-end read technology offers a convenient and reliable method of detecting genome structural rearrangements, effectively bypassing an intensive search for non-homologous breakpoints. In the mapping step, paired-end coordinates are recorded for each read, which we filtered using simple text edit scripts. We used utilities of the SAMtools framework to quantify all paired-end reads that mapped to different chromosomes (mismatches). Further NUMTs were filtered from the mismatch output files, where one paired end mapped to mtDNA and the other to a nuclear coordinate. Since breakpoints were not precisely identified, we used the paired-end coordinate as a proxy for nuclear insertion site because the average distance between properly mapped reads was short (ca. 150 bp).

In addition to challenges presented by NUMT detection, we recognized challenges related to data normalization that are not addressed by traditional copy-number analyses. NUMTs represent a subset of the mapped genomic read count, and local representation cannot be assumed constant across tumor genomes. To study the NUMT abundance, we defined a metric for calculating relative NUMT abundance from high-throughput genomic sequence data. First, relative NUMT abundance was normalized to mapped read count across a given genomic region, expressed as a ratio $\left(P_{i j}\right)$ :

$$
P_{i j}=\frac{N_{i j}}{M_{i j}}
$$

Let $N_{i j}$ and $M_{i j}$ represent NUMT read count and mapped read count within a genomic interval $(i)$, respectively, for genome type $(j)$ for an individual; where a 
genomic interval is defined as whole genome (gen), chromosome (chr), chromosome arm (arm), or 2.5-mbp sliding window (win); and where genome type $(j)$ is either tumor $(t)$ or matched blood-derived healthy $(h)$ genome of an individual. The change in tumor and matched healthy NUMT proportions is defined as $\Delta P_{i}=P_{i t}-P_{i h}$ and the ratio is given as:

$$
R_{i}=P_{i t} *\left(P_{i h}\right)^{-1}
$$

where $R_{i}$ represents the proportional fold-change in NUMT abundance within a genomic region $(i)$ for tumor $(t)$ versus matched blood-derived healthy $(h)$ genomes. When sub-genomic regions (e.g., sliding windows and cytobands) were compared, $P_{i h}$ was replaced with $\bar{P}_{i h}$, where $\bar{P}_{i h}$ is the average NUMT proportion within the genomic interval for all blood-derived healthy samples sharing the same plate barcode to avoid zero denominator because blood-derived healthy samples may not have any NUMTs within the short regions.

The $R_{i}$ derived earlier can be less than or greater than 1 due to the asymmetric property of ratios (e.g., where $R_{i}=0.5$ represents a change in the opposite direction, i.e., there are twice as many NUMTs in healthy samples compared to tumor; and similarly, $R_{i}=2.0$ represents a change in the positive direction, i.e., there are twice as many NUMTs in tumor samples compared to their matched blood-derived normal samples). In order to compare NUMTs between tumor and blood-derived healthy samples within the genomic regions, it is necessary to rescale $R_{i}$ values less than one and to collapse values of one and negative one to zero. Further, we defined an indicator value $S_{i}$ to denote the direction of change between tumor and matched blood-derived normal NUMT abundance:

$$
S_{i}=\frac{\Delta P_{i}}{\left|\Delta P_{i}\right|}
$$

and:

$$
R_{i}^{\prime}=R_{i}^{S_{i}} * S_{i}
$$

Let the $R_{i}^{\prime}$ represent the rescaled proportional foldchange in relative NUMT abundance between tumor and matched blood-derived normal samples. $R_{i}^{\prime}$ was calculated for the nuclear genome using five nested data partitions, where $i$ represents whole genomes (gen), chromosomes (chr), chromosome arms (arm), cytobands $(c y t)$, and sliding windows (win). For nuclear genomescale comparisons, normalization to mapped reads $\left(M_{i j}\right)$ did not include sex chromosomes due to sequence representation bias associated with differences in chromosome length. For small-partition comparisons (e.g., sub-band), samples were pooled before normalization to avoid bias associated with zero denominators. For the mitochondrial genome, $R_{i}^{\prime}$ was calculated for three nested data partitions, where $i$ represents genome $(m t g)$, replication strand $(m s t)$, and gene (mgn).

The relationship between $R_{\text {arm }}^{\prime}$ and mapped read count was evaluated with linear regression to assess whether NUMT transposition is coincident with aneuploidy. The relationships between $R_{w i n}^{\prime}$ were evaluated between GC content to assess whether transcriptional activity predisposes the nuclear genome to integration of non-homologous DNA. All statistical analyses were conducted in the $\mathrm{R}$ statistical environment (http:// www.R-project.org/). The NUMT proportions and abundance in blood-derived normal and primary tumor sites are quantified in Additional file 1: Table S1.

\section{YME1L1 gene knockout and NUMT analyses in isolated nuclear DNA}

Using the CRISPR-Cas9 method, we knocked out the human YME1L1 gene in human breast epithelial MCF-7 cells as described earlier [46]. To avoid any mtDNA contamination in NUMT analyses, we isolated nuclear fractions free of mitochondrial contamination. Briefly, YME1L1 knockout and wild-type MCF-7 cells were lysed using lysis buffer (10 mM HEPES, pH 7.9, $10 \mathrm{mM} \mathrm{KCl}$, $0.1 \mathrm{mM}$ EDTA) containing 10\% IGEPAL detergent for $10 \mathrm{~min}$ at room temperature and centrifugation at $15,000 \mathrm{xg}$ for 3 minutes was carried out to pellet the intact nuclei. To completely avoid cytoplasmic fraction contamination in the nuclear pellet, the lysis step was repeated one more time. The purity of the nuclear fraction was ascertained by performing western blotting for mitochondrial encoded cytchrome oxidase II (COXII) protein. Nuclear DNA was prepared from the nuclear pellet using the NAOH boiling method [47]. Mitochondrial DNA content in the nuclear fraction was analyzed by real-time PCR by absolute quantification using primers for COXII (mtDNA-encoded gene) and Beta-2 microglobulin (B2M; nuclear DNA-encoded gene). B2M served as an internal control.

\section{Yeast transformation and genetic selection}

Yeast expression vectors (pYX113, pPT31-yYme1, and pYX113-hYme1L) were transformed using the lithium acetate, single-stranded DNA, polyethylene glycol method [48-50]. Following transformation, yeast harboring the desired vector was selected using synthetic dropout (SD) medium $(0.67 \%[\mathrm{w} / \mathrm{v}]$ nitrogen base without amino acids, $0.07 \%$ [v/v] drop-out amino acid mix (-His/-Trp/-Ura), 0.02\% [w/v] L-histidine and excluding the amino acid that is a selectable marker, $2 \%[\mathrm{w} / \mathrm{v}] \mathrm{dex}-$ trose, and $1.5 \%$ agar for agar plates). Single cell colonies from plates lacking uracil were cultured in glucose medium and $1 \times 10^{4}$ and $5 \times 10^{7}$ or $5 \times 10^{8}$ cells were 
plated in triplicate onto YPD and SD media lacking tryptophan.

The yeast strains throughout this study were grown in YPD medium $(1 \%[\mathrm{w} / \mathrm{v}]$ yeast extract, $2 \%[\mathrm{w} / \mathrm{v}]$ bactopeptone, $2 \%[\mathrm{w} / \mathrm{v}]$ dextrose) or YPG medium $(1 \%[\mathrm{w} / \mathrm{v}]$ yeast extract, $2 \%[\mathrm{w} / \mathrm{v}]$ bacto-peptone, $3 \%[\mathrm{w} / \mathrm{v}]$ glycerol, $\mathrm{pH} 4.9)$ at $30^{\circ} \mathrm{C}$. The yeast strains constructed and used in this study are detailed in Table 1. The yeast Yme1-1 strain (PTY62) was transformed with a plasmid expressing the yeast YME1 (yYme1) or human YME1L1 (hYme1L1) gene under the alcohol dehydrogenase $(\mathrm{ADH})$ promoter.

\section{Results}

\section{Adenocarcinoma genomes contain increased NUMTs compared to healthy genomes}

Since mitochondrial abnormalities are frequently described in cancer, we asked whether mitochondrial dysfunction results in increased prevalence of NUMTs in tumors. Constitutively, tumor genomes contained more NUMTs than matched blood-derived healthy genomes when normalized to mapped read pairs. When scanned for NUMT proportion and distribution on a genomewide scale across 57 samples, tumor genomes contained, on average, 4.42-fold more NUMTs than healthy normal genomes. Box plots (Fig. 2a, b) indicate the mean, dispersion, and skewness of NUMT density for the genome groups. Although both tumor and matched normal genomes show right-skewed distributions (Fig. 2c), the third quartile $\left(3.43 \times 10^{-6}\right)$ of the normal blood genome group is less than the first quartile (3.13E-6) of the tumor genomes, indicating significant difference in the NUMT density. A one-tailed, paired $t$-test was performed on tumor and matched blood-derived healthy samples to determine the statistical significance $\left(P\right.$ value $\left.8.79 \times 10^{-13}\right)$ of the $\log$ transformed NUMT abundance levels. In one case, however, 22-fold more tumor NUMTs were observed in a Caucasian woman. This individual's vital status was pronounced as deceased after reporting her pathologic tumor stage as I. These studies suggest that colorectal adenocarcinomas contain increased NUMTs compared to blood-derived healthy NUMTs.

\section{NUMT abundance in blood correlates with NUMT abundance in tumor}

Increasingly, molecular characteristics of tumors are demonstrated to be reflected in the blood of cancer

Table 1 Sacharomyces cerevisiae strains used in the study

\begin{tabular}{lll}
\hline Strain & Genotype & Mitochondrial genotype \\
\hline PTY62 Yme1-1 & MATa ura3-52 lys2 leu2-3, & $\rho+($ TRP1) \\
& 112 trp1-41 yme1-1 & \\
PTY62-yYme1 & & \\
PTY62-hYme1L1 & \\
\hline
\end{tabular}

patients. We therefore envision that increased NUMT incidence observed in tumors may be found in the matched blood of cancer patients. In order to determine the predictability of tumor NUMT density based on the NUMT frequency in matched blood-derived healthy samples, a simple linear regression was performed on the log base 2-transformed proportions of NUMTs in blood-derived healthy genomes (Pgenh) and tumor genomes (Pgent). Linear regression revealed a positive relationship between Pgent and Pgenh with an $\mathrm{R}^{2}=0.17, P$ value $=0.0016$ (Fig. 3a, b). These results indicate that there is a positive correlation between proportions of NUMTs in blood-derived and primary tumor in colorectal cancer samples.

\section{Colorectal tumors in women harbor more NUMTs}

Recent studies suggest hormonal regulation of mitochondrial functions [51]. Therefore, we delineated NUMT distribution among males and females. Colorectal tumors of women harbored more NUMTs than those of men (Fig. 4). Women had a median NUMT fold change proportion of 4.52 compared 3.1 for men. We conclude that tumors from women contained more NUMTs than those from men.

\section{NUMT abundance is associated with patient survival}

It is conceivable that increased NUMT insertions may confer metastatic disease resulting in the death of patients. Therefore, we determined NUMT abundance and correlated it with patient survival. Figure 5 demonstrates that the NUMT abundance is amplified many fold in deceased individuals. Based on the survival rate among both sexes and vital status, there appears to be corroborating evidence that NUMTs in colorectal tumor genomes of women have a tendency to be amplified and are associated with patient survival. However, further analyses with larger sample sizes are required to make a definite conclusion. When overlaying these findings with circos integrators (Fig. 6; Additional file 2: Figures S1; Additional file 3: Figure S2), three of the four deceased individuals were reported to have pathologic tumor stage IIIC and IVA. Overall, except for one individual (women, stage I, deceased status), these results suggest that NUMT abundance increases with tumor grade and is significantly amplified in women.

\section{NUMT abundance positively correlates with GC content and gene density}

GC content is positively correlated with gene density; hence, regions of high $\mathrm{GC}$ content have higher relative gene density compared to regions of low GC content [52]. Also, as Giemsa-negative chromosomal bands are gene-rich regions of DNA compared to bands positive for Giemsa staining [53], we extended our NUMT analyses 


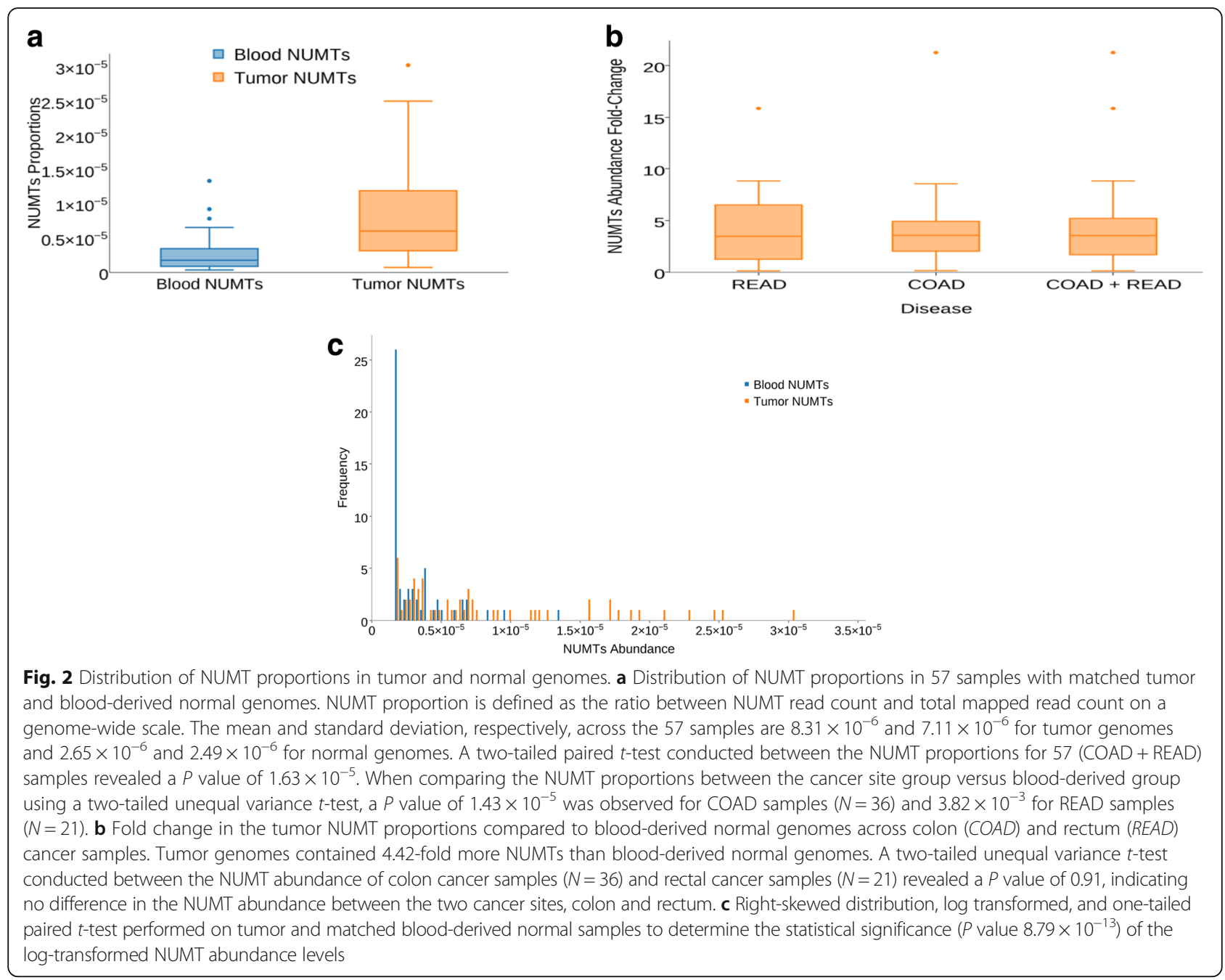

to the chromosomal cytobands. Our study demonstrates a positive relationship between NUMT abundance and GC content of gene-rich regions, having an abundance foldchange of 4.2 or more (Fig. 7).

Overall, among the groups that had a fold change of 2 or more in NUMT abundance, although the low GCcontent cytobands gpos50 and gpos100 were at the top of the NUMT abundance list for chromosomal cytoband windows, GC-rich "gneg" regions were prominent among cytobands exhibiting more than a 4.2-fold change in NUMT abundance. This pattern observed for the outlier data above 4.2 fold-change of NUMT abundance within GC-rich regions of gneg clearly shows a strong correlation between elevated NUMT abundance and GC content. Based on Fig. 7, as indicated by data points above the third quartile level, gneg regions are accountable for the greater than normal NUMT abundance, indicating a positive correlation between gene density and NUMT abundance. A previous study has shown that gpos100 sub-bands are also enriched with LINEs [54], and other researchers have demonstrated secondary amplification via LINE transposition to be an important mechanism of NUMT accumulation $[11,20]$. Our results corroborate the empirical evidence. Clearly, a great deal of new research is needed to elucidate the causes and consequences of NUMT transposition. We hope that our discovery will serve as an impetus for such inquiry.

\section{Mitochondrial fragile sites associated with NUMTs}

To identify fragile sites in the mitochondrial genomes that act as hotspots for mtDNA to immigrate into the nuclear genome, paired-end reads with one end mapping to the mitochondrial genome and the other end to the nuclear genome, NUMTs were further investigated. As illustrated in Fig. 8, fragile sites were identified within complex I (ND1) and complex IV (MT-CO1/COX1, COX III) mitochondrial regions, which are known for harboring mutations in different cancer sites. Based on the migration pattern of NUMTs, breakpoint sites in 

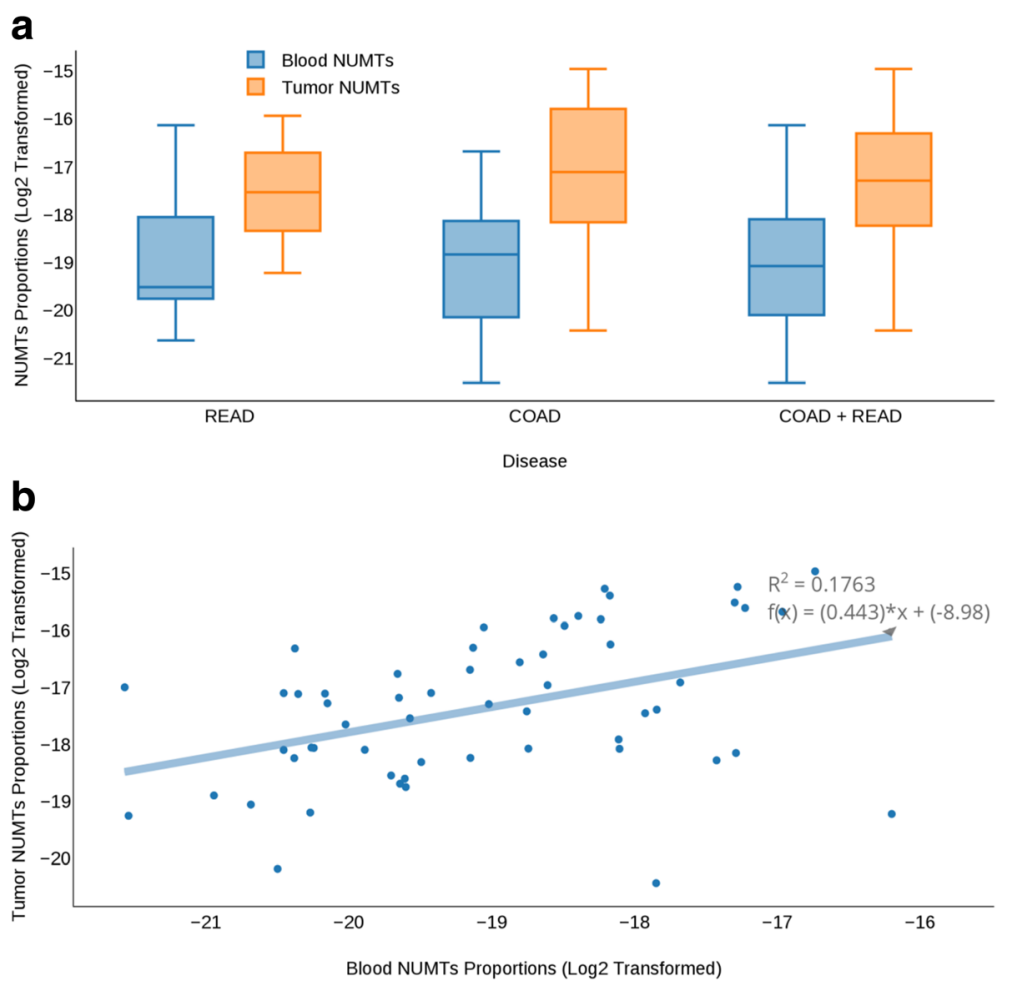

Fig. 3 Log2-transformed NUMT proportions in tumor and blood-derived normal (Pij) genomes. a Log2-transformed NUMT proportions in tumor and normal genomes showcasing the difference in their means, indicating higher NUMT distribution in tumor genomes compared to matched blood-derived normal genomes. A two-tailed paired t-test conducted between the log2-transformed NUMT proportions for 57 samples revealed a $P$ value of $1.87 \times 10^{-11}$, showing significant difference in the log-transformed NUMT proportions between primary tumors and blood-derived normal genomes. $\mathbf{b}$ Relationship between abundance measures of blood-derived normal genome NUMTs and tumor genome NUMTs showing a positive relationship with $\mathrm{R}^{2}=0.17$ and $P$ value $=0.0016$

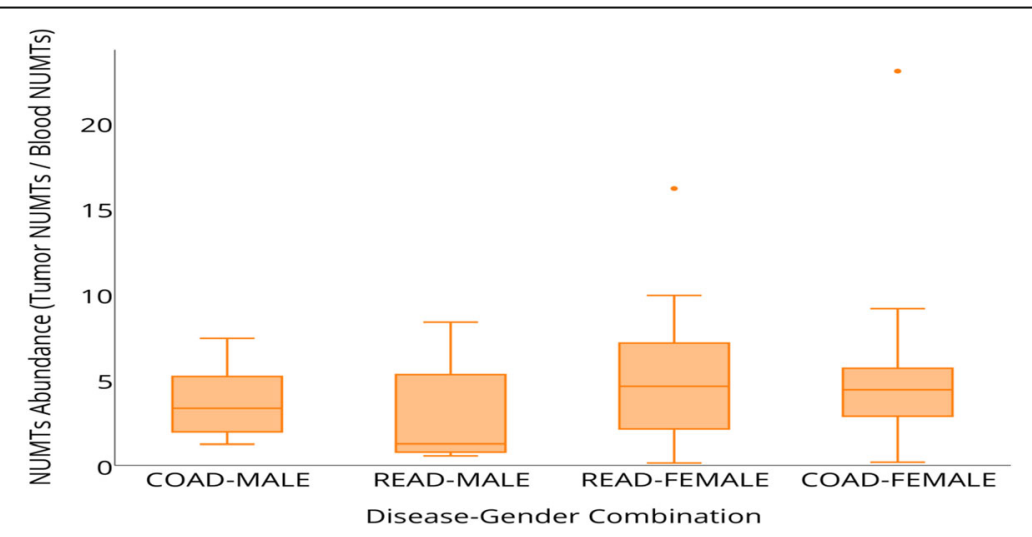

Fig. 4 NUMT abundance across disease-sex combination. Colorectal tumors from women have higher NUMT abundance proportion (tumor NUMT/ blood normal NUMT) than those from men. Women had a median NUMT fold change abundance of 4.52 (range 0.11 to 22.9 ) compared 3.1 for men (range 0.53 to 8.3). COAD colon adenocarcinoma, READ rectum adenocarcinoma. To investigate the sex difference in the NUMT abundances, an unequal variance $t$-test was performed on the raw NUMT proportions observed by the members of the two sex groups stratified by "blood-normal" and "primary tumor" classifications. The $P$ value was 0.03 between males $(N=23)$ and females $(N=34)$ for blood-derived normal samples and 0.08 for tumor samples 


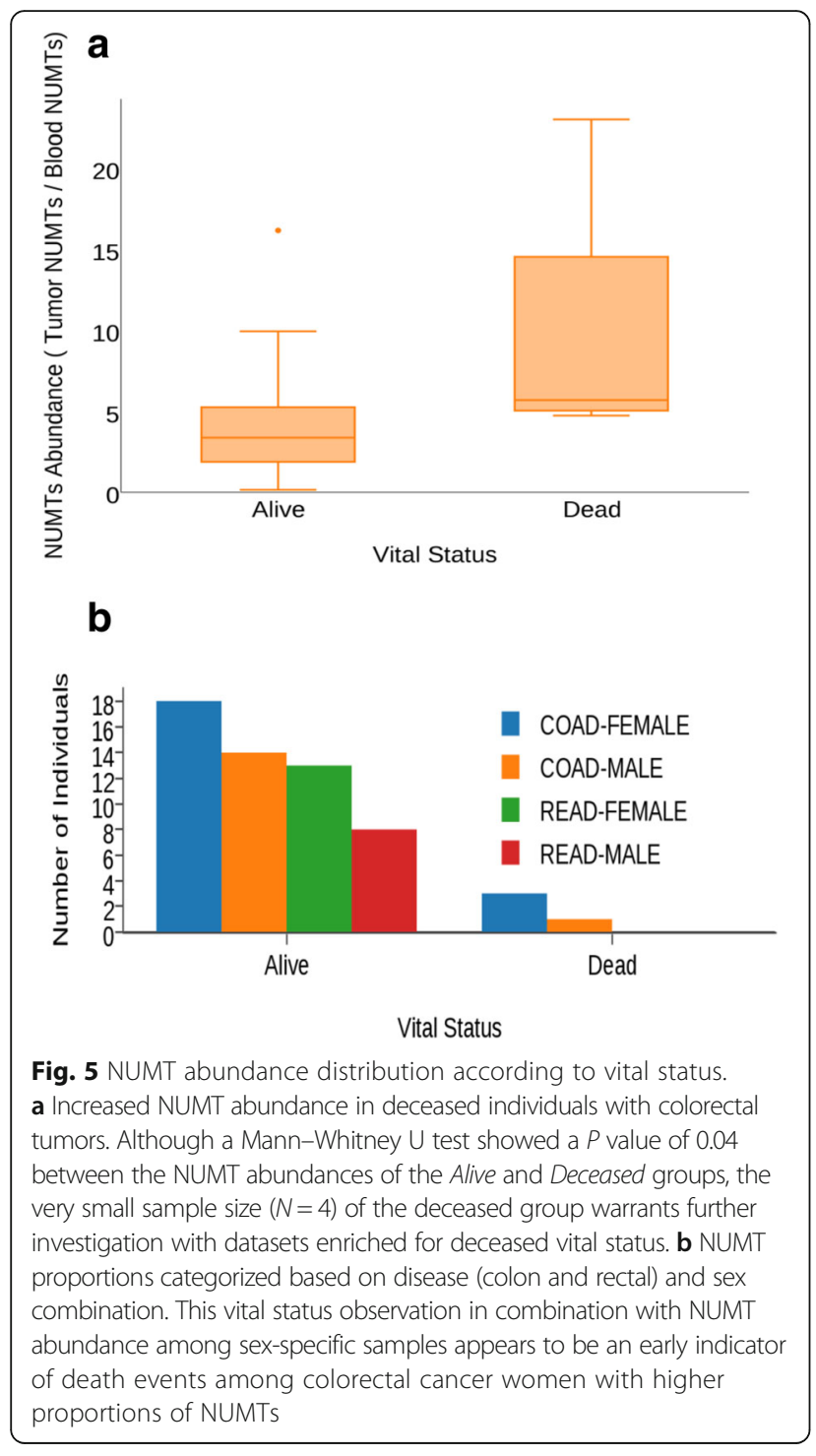

COX1 and ND1 are responsible for numtogenesis in the nuclear genome of colorectal cancer.

\section{YME1L1 inactivation leads to increased numtogenesis} In the yeast Saccharomyces cerevisiae, YME1 is reported to be an important suppressor of mtDNA migration to the nucleus [55]. Interestingly, the YME1L1 gene encodes the human homologue of yeast mitochondrial AAA (ATPases associated with diverse cellular activities) metalloprotease, Yme1p. YME1L is a functional homologue of Yme1p, with conserved roles in mitochondrial assembly, integrity, and DNA metabolism [56]; however, its function in suppressing NUMTs is not known.

We conducted in silico YME1L1 mutation analysis in all colorectal cancer cases $(n=57)$ used for NUMT analysis in this study. We determined that of $57 \mathrm{CRC}$ tumors, $\sim 16 \%$ contained mutations in YME1L1. A total of 24 mutations (five exonic, 17 intronic, and two in the 3'
UTR) in YME1L1 were identified. All five exonic mutations were frameshift mutations (Fig. 9a, b).

We expanded our analysis of Yme1L1 in TCGA database. This analysis revealed a high incidence of Yme1L1 mutations in CRC (Fig. 9c). The mutations in Yme1L1 include missense and synonymous substitutions and inframe and frameshift deletions (Fig. 9c). Most of the mutations in Yme1L1 in human colorectal cancer fall into two categories: missense substitutions ( 68\%) and synonymous substitutions $(20 \%)$. The relative distribution of various mutations is summarized as a pie chart in Fig. 9c. We also analyzed Yme1L1 mutations in other human cancer types and observed a high mutation frequency in all the tested cancer types (Fig. 9d).

We determined whether inactivation of the human YME1L1 gene increases NUMT formation. For this, we created a YME1L1 knockout in a human cell line using YME1L1 gene-specific CRISPRs. We prepared nuclear fractions free of mitochondrial contamination and quantified the amount of mtDNA present in the nuclear fraction of these cells. We observed a strikingly increased amount of mtDNA in the nuclear fraction of YME1L1 knockout cells compared to wild-type cells (Fig. 9e). These results identify YME1L1 as the first NUMT suppressor gene in humans and suggest that inactivation of YME1L1 leads to increased numtogenesis.

\section{Human homologue of YME1 suppresses migration of mtDNA to the nucleus}

We asked whether the phylogenetically conserved role of human YME1L1 can rescue the migration of mtDNA in a yeast strain in which $Y M E 1$ is disrupted. We utilized the Yme1-1 yeast strain, which harbors a mutation which leads to inactivation of the $Y M E 1$ gene [57]. In this strain, the auxtotrophic endogenous nuclear TRP1 gene is deleted and inserted into the mitochondrial genome. Since the required transcription machinery for TRP1 is only present in the nucleus, the mitochondrially inserted TRP1 gene is only functional when it migrates to the nucleus, permitting analysis of mtDNA migration to the nucleus $[57,58]$.

To determine whether human Yme1L1 is expressed in the Yme1-1 strain, western blotting was performed. Indeed, hYme1L1 was expressed in the Yme1-1 hYme1L1 strain (Fig. 9f). When the Yme1-1 vector was plated under tryptophan selection, it showed a significantly large number of colonies (Fig. 9g). These data support a previous observation that migration of mtDNA to the nucleus is high in Yme1-1 cells [57]. Yme1-1 cells expressing yeast Yme1 (yYme1) display only a few $(<50)$ tryptophan-positive colonies, suggesting that accumulation of mtDNA fragments in the nucleus in the Yme1-1 strain was prevented to a greater degree by re-introducing 


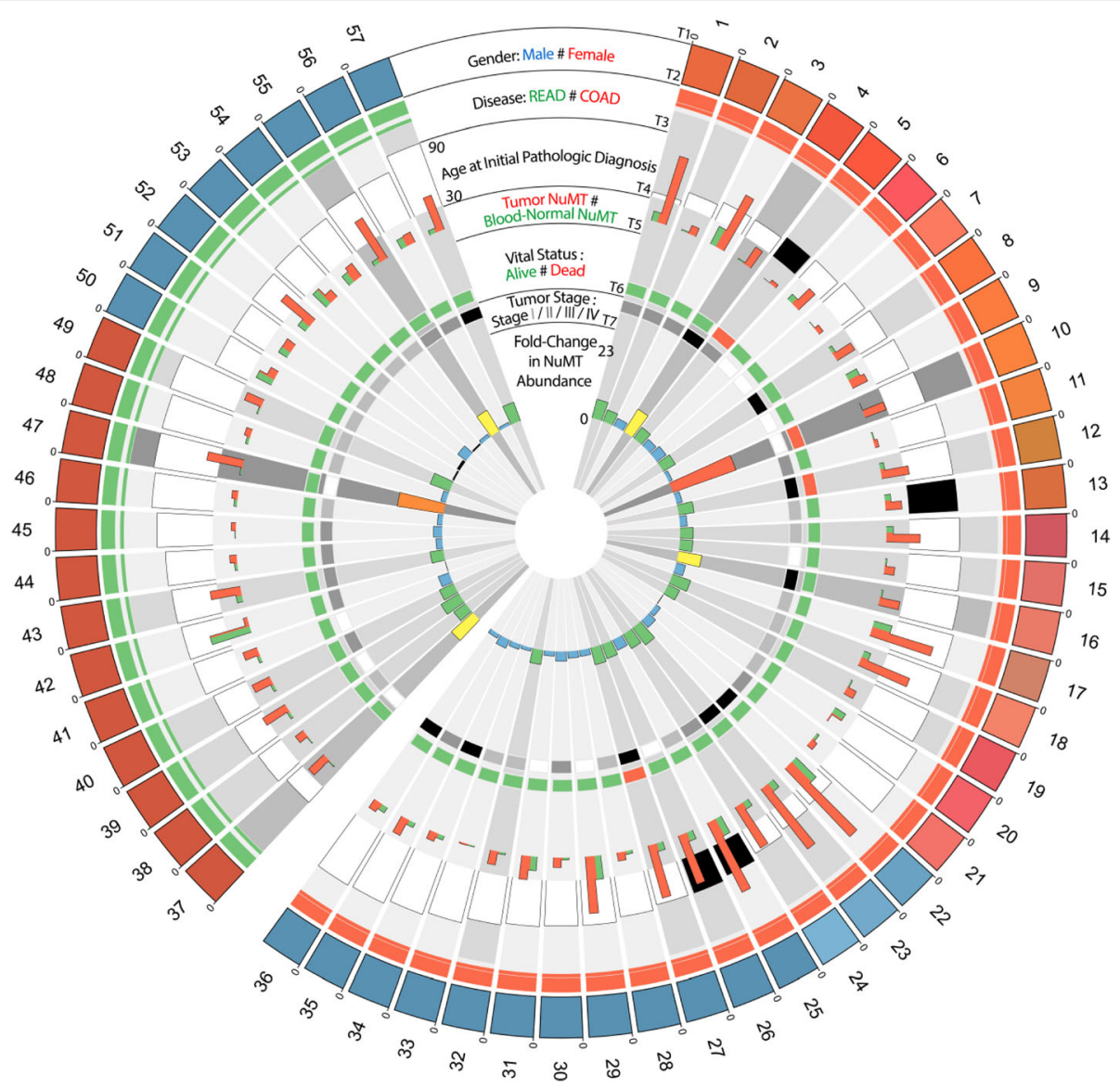

Fig. 6 NUMT density in tumor and normal genomes (sorted by disease (T2), sex (T1), and age at initial pathologic diagnosis (T3)). Each peripheral node represents a TCGA sample whose blood-derived normal and tumor genomes were used in this study. From the outside to inside, tracks are ordered from 1 to 7 (T1-T7). T1: Sample sex where red nodes represent female and blue nodes represent male. T2: Disease type information. Rectal adenocarcinoma (READ) is rendered as green bands and colon adenocarcinoma (COAD) as red bands. T3: Age at initial pathologic diagnosis ranging from 30 to 90 years. White and black filled bars represent white and black race, respectively. T4: Red columns represent NUMT proportion in tumor genomes and green columns represent blood-derived normal NUMT proportion. T5: Vital status of the patients where red indicates deceased individuals and green alive status. T6: Stage of tumor represented in grey-scale—stage I white, stage II grey, stage III dark grey, and stage IV black. T7: Fold-change in NUMT abundance. Samples at <1-fold are rendered as colored bands; 1-4-fold, blue; 4-8-fold, green; 8-12-fold, yellow; 12-20-fold, orange; and >20-fold, red

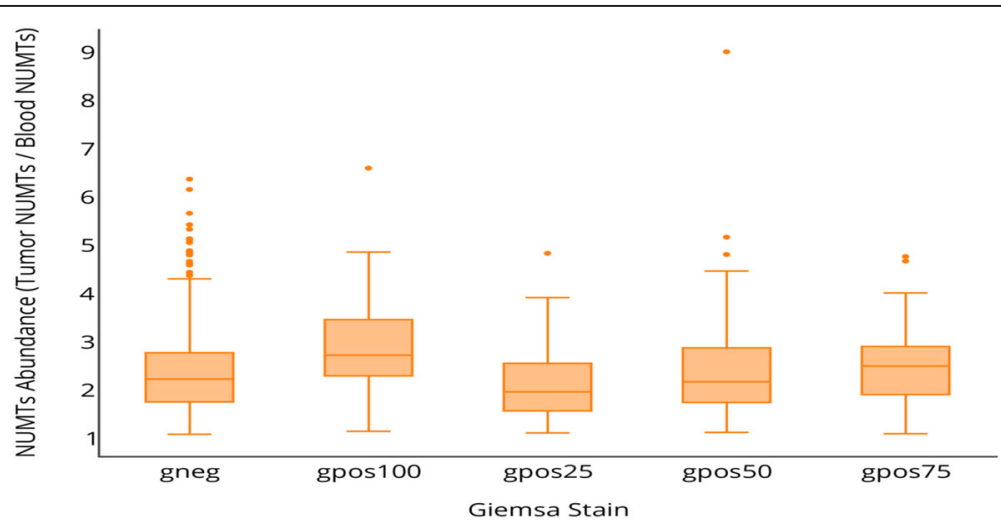

Fig. 7 Correlation of NUMT abundance and GC content. Positive correlation between gene density and NUMT abundance. GC content is positively correlated with gene density; hence, regions of high GC content have higher relative gene density than regions of low GC content. Sample sizes (number of chromosome bands annotated by a certain Giemsa stain value) for the various Giemsa stain groups are as follows; gneg, $N=366 ;$ gpos 100, $N=75 ;$ gpos25, $N=73 ;$ gpos50, $N=109 ;$ and gpos $75, N=82$ 


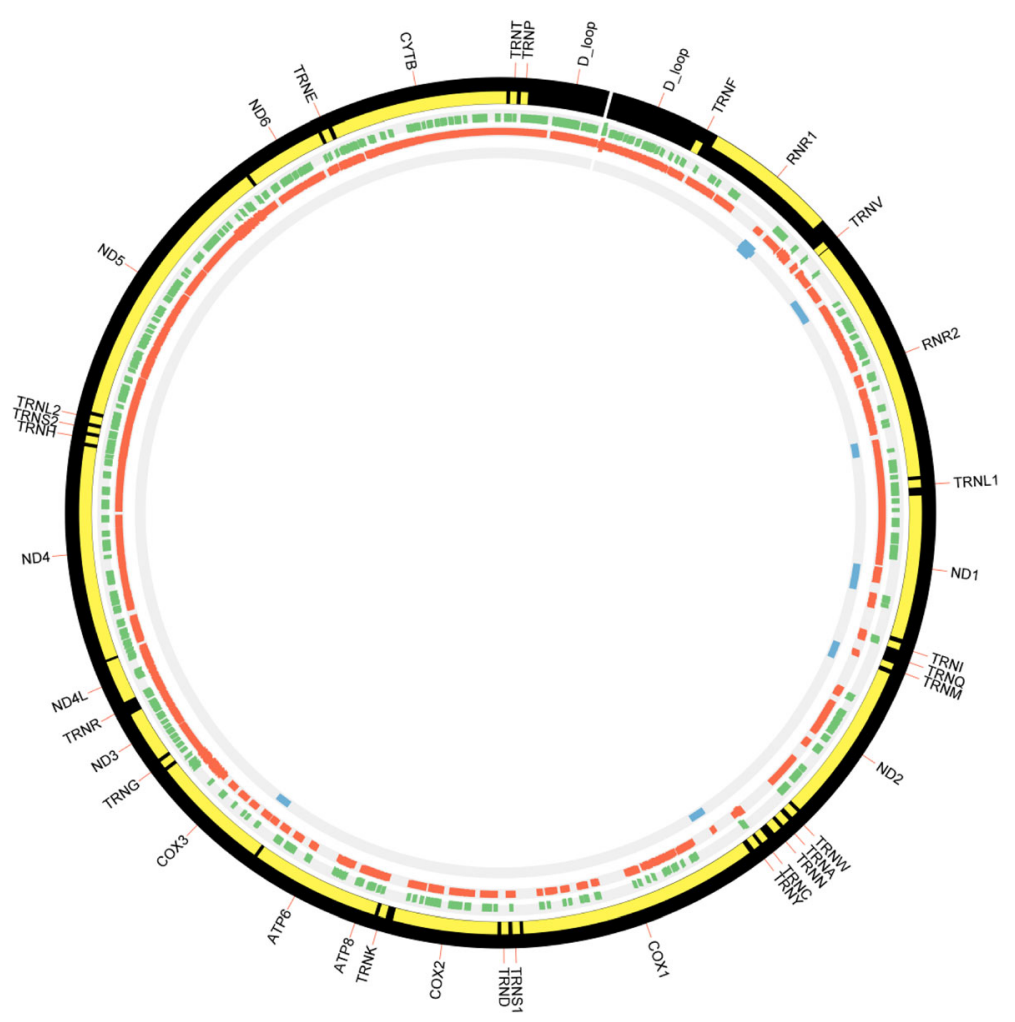

Fig. 8 Top fragment sites of the mitochondrial genome identified in the nuclear genome. Genes on the mitochondrial genome and their potential fragile sites involved in the process of numtogenesis. Bands from the outside to inside represent: mitochondrial gene names; gene segments; numtogenesis regions of blood-derived normal samples (green bands); numtogenesis regions of primary tumor samples (red bands); numtogenesis regions unique to tumor samples but not observed in blood-derived normal samples (blue bands)

yYme1 (Fig. 9g). The same number of Yme1-1 cells harboring hYme1L1 also produced significantly low number of tryptophan-positive colonies compared to Yme1-1 cells (Fig. 9g). However, the number of typtophan-positive colonies in this case was a little higher $(>100)$ compared to yYme1-expressing cells. This suggests that hYme1L1 can partially rescue the phenotype of mtDNA escape in Yme11. We conclude that hYme1L1 suppresses migration of mtDNA to the nucleus.

\section{Discussion}

Numtogenesis, a natural phenomenon leading to migration of mitochondria, mitochondrial proteins, mtRNA, or mtDNA into the nucleus, is an ongoing cellular process reported in eukaryotic cells [2-9]. Although the occurrence of NUMT and the phenomenon of numtogenesis have been reported, its role in cellular and organismal function and in human health and disease remains relatively unexplored. Our study revealed that somatic NUMTs are frequently found in colorectal cancer. Consistent with our finding, two previous studies have associated NUMTs with carcinogenesis; one that found NUMTs containing LINEs in rat and mouse tumors [20] and a study of a cervical carcinoma cell line [19].
We provide evidence for increased NUMT insertions in the nuclear genomes of colorectal adenocarcinomas relative to matched control samples. NUMT occurrence was influenced by pathological cancer stage and sex between tumor and control groups. Germ line NUMT insertions leading to diseases have been identified [59]. These diseases include severe plasma factor VII deficiency and bleeding diathesis [60], mucolipodosis IV [61], Usher syndrome [62], and a rare Pallister-Hall syndrome [63]. These NUMT insertions were found in coding genes. NUMT insertions in these genes can reduce cellular fitness, leading to cellular dysfunction-induced cell death, which may underlie these human diseases [64]. Conceivably, somatic NUMT insertion in tumor suppressor gene(s) may disrupt pathways which can contribute to tumorigenesis. Similarly, NUMT may activate oncogene(s) involved in tumor development. Indeed, integration of mtDNA fragments in the MYC locus in HeLa cells [65] and in the nuclear genome of mouse embryonic fibroblasts [66] has been identified. It appears that the integration of mtDNA in the nuclear genome of mouse embryonic fibroblasts led to the malignant transformation [66].

Increased somatic NUMT insertion in the nuclear genome of tumors may be associated with mitochondrial 


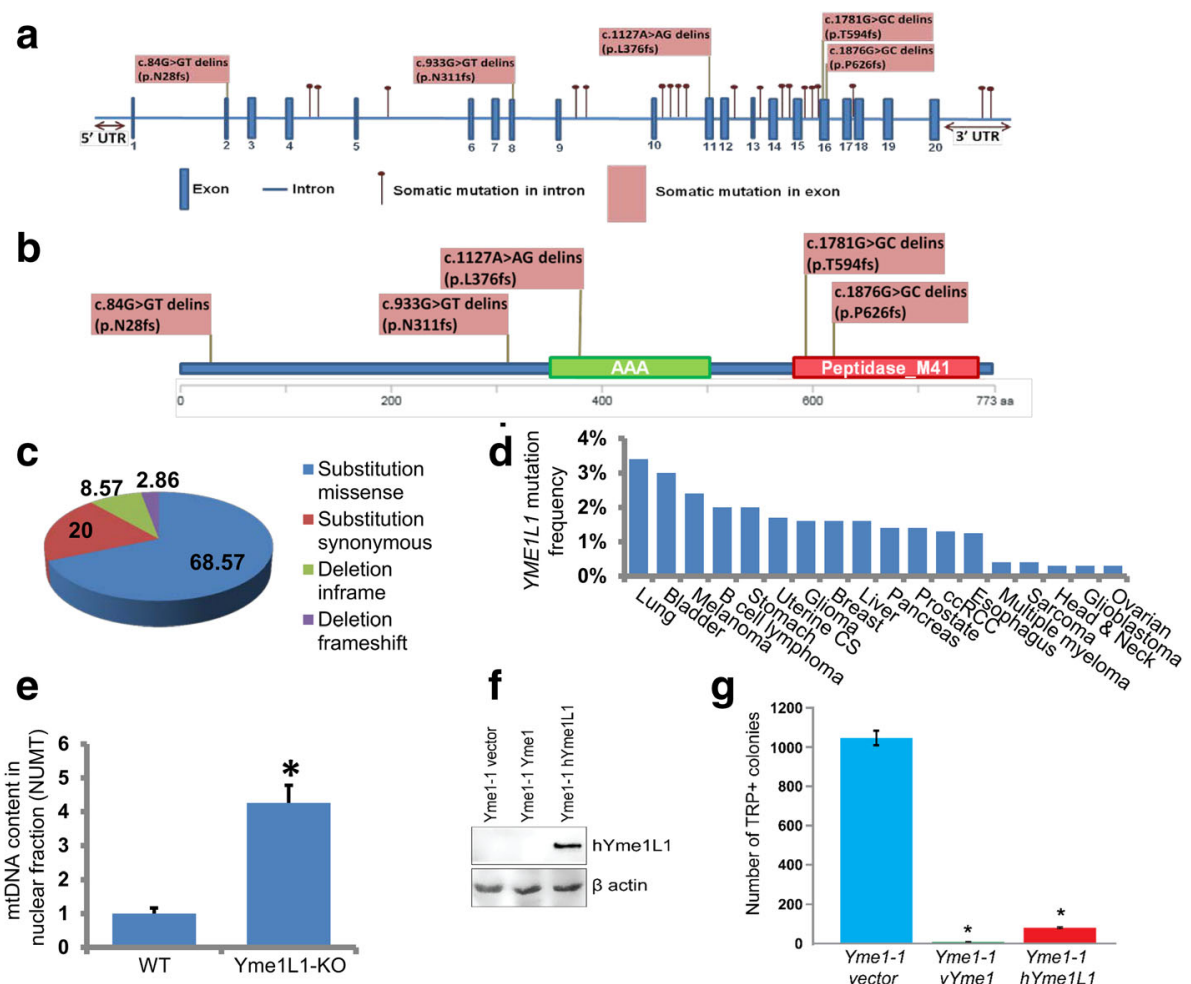

Fig. 9 Human YMEIL1 inactivation leads to increased numtogenesis. TCGA tumor sample data used in this study were screened for mutations in the YME1L1 gene. A total of 24 mutations (five exonic, 17 intronic, and two in the 3' UTR) in YME1L1 were identified. All five exonic mutations were frameshift mutations. a, b The position of these mutations in the YME1L1 gene (a) and protein (b). c The total colorectal cancer samples available in TCGA database were analyzed on 4 April 2016 for mutations in Yme1L1 and their types determined. d Mutations in Yme1L1 in other cancers were also analyzed using the cBioPortal database. Altered frequency of Yme1L1 mutations in different cancer types are represented. e mtDNA content in nuclear fractions, i.e., NUMT accumulation was analyzed in wild-type (WT) and YME1L1 knockout (Yme1L1-KO) human cell lines. NUMT accumulation was about fourfold increased in YME1L1-KO cells compared with wild-type cells. Data are expressed as mean \pm standard error of the mean (sem); ${ }^{*} P<0.05$, Student's t-test. $\mathbf{f}$, g The yeast PTY33-Yme1-1 $(\rho+$, TRP1) strain was transformed with empty plasmid and plasmids expressing yYme1 and hYme1L1 with URA marker as indicated. Transformed cell colonies were selected by synthetic dropout medium lacking URA. $\mathbf{f}$ Whole cell lysate from the Yme1-1 vector, Yme1-1 yYme1, and Yme1-1 hYme1L1 strains was subjected to SDS-PAGE and western blotting was performed with antibodies against hYme1L1 and $\beta$-actin. g Yme1-1 vector, Yme1-1 yYme1, and Yme1-1 hYme1L1 cells were spread on plates lacking tryptophan; the experiment was performed three times in triplicate. Data are expressed as mean \pm sem; ${ }^{*} P<0.05$, Student's $t$-test

dysfunction. Mitochondrial dysfunction is a consistent feature of a variety of tumors and is described to be a hallmark of cancer [67-72]. We have previously demonstrated that mitochondrial dysfunction induces genomic instability in the nucleus [30,31,33]. However, genetic instability associated with mitochondrial dysfunction has been described to be point mutations or chromosomal aneuploidy [73-75]. The nuclear genome instability was induced due to increased oxidative stress caused by the changes in the nucleotide pool [30]. Hadler et al. [76] proposed "a unitary hypothesis for carcinogenesis", speculating that a breakdown of mito-nuclear symbiosis leads to development of cancer; they suggested release of mtDNA due to damage to the mitochondrial membranes. Using a sliding window approach, we determined NUMT insertion in tumor genomes. When we searched for the origin of these nuclear NUMT landing sites among colorectal cancer samples, we found three potential fragile sites within the mitochondrial genes ND1, COX1, and COX3, whose association with colorectal cancer has already been well investigated [77, 78]. Interestingly, in most females NUMTs originated from the same mitochondrial regions while in males NUMTs originated from different regions of mtDNA.

In the yeast $S$. cerevisiae, mitochondrial dysfunction leads to increase escape of mtDNA into the nucleus [57]. Mutations in the $Y M E 1$ gene induce migration of mtDNA to the nucleus [57]. Yme1p is a multifunctional protein, controlling mitochondrial quality, which plays an important role in mitochondrial biology, including assembly of mitochondrial respiratory complexes, and importantly in mitophagy $[79,80]$. We identified that $16 \%$ of analyzed colorectal tumors contained mutations in the YME1L1 gene (Fig. 9a, b). Mutations in Yme1L1 were also found in a variety of other types of tumors (Fig. 9d). We demonstrate that the human homolog of 
yeast Yme1 functions as a "NUMT suppressor". Human YME1L1, when expressed in a mutant Yme1 yeast strain, reduces the migration of mtDNA to the nucleus (Fig. 9g). These observations support YME1LI as a NUMT suppressor gene in humans whose inactivation leads to increased numtogenesis. Yme1 removes the damaged or dysfunctional mitochondria by mitophagy and maintains a healthy pool of mitochondria in the cell [81]. These observations implicate mitophagy in mediating accumulation and transfer of mtDNA into the nuclear genome. These observations lead us to suggest that the mechanism underlying numtogenesis may involve Yme1-mediated mitophagy. Mitophagy is a stringent mechanism that controls the quality of mitochondria in cells [81]. Compromised mitophagy due to loss of Yme1 [80] or an acid endonuclease DNase II $\alpha$ [82] can lead to accumulation of incompletely digested mtDNA in the cytoplasm that ultimately ends up in the nucleus (Fig. 10).

It is conceivable that a direct physical association or fusion between the mitochondrial and nuclear membranes and encapsulation of mitochondria in the nucleus $[83,84]$ may contribute to numtogenesis. Observations supporting encapsulation of mitochondria in the nucleus have been reported [2-5, 7-9]. The nuclear envelope breaks down during mitosis, leading to disruption of the physical barrier seperating the nucleoplasm and cytoplasm [85]. This stage of the cell cycle can provide an opportunity for mitochondria to enter into the nucleus. Furthermore, cancer cells often exhibit a ruptured nuclear envelope [86]. Decreased expression of lamins, important constituents of the nuclear membrane, contributing to

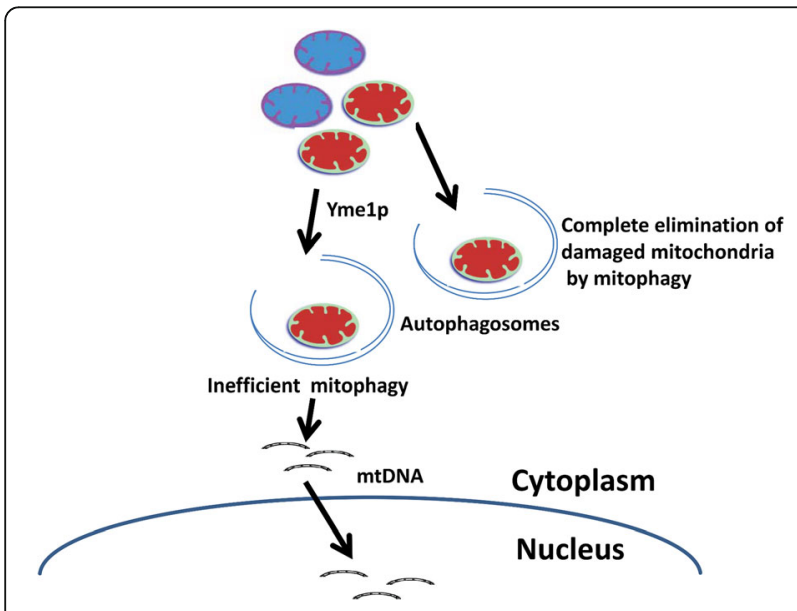

Fig. 10 Mechanism underlying numtogenesis. Our observations support the role of Yme1 in numtogenesis. The role of Yme1 in the regulation of mitophagy is well established. Mitophagy is a stringent mechanism that controls the quality of mitochondria in cells by degrading dysfunctional mitochondria. Compromised mitophagy due to altered Yme1 function leads to accumulation of undigested mtDNA in the cytoplasm that ultimately ends up in the nucleus, a process we have named numtogenesis nuclear rupture in cancer cells, has also been reported [87]. Lamins in the nuclear membrane bind to chromatin and hold chromosomes in place and thus reduce chromosome breakage [88]. Indeed, patients with laminopathy resulting from reduced lamin expression contain mitochondria in the nucleus. It is likely that loss of lamin expression in cancer cells helps migration of mitochondria into the nucleus, resulting in eventual integration of mtDNA into the nuclear genome. This phenomenon might be a survival mechanism for cancer cells [13].

It is unclear how numtogenesis alters the nuclear genome functions. Tsuji and coauthors [25] hypothesized that the underrepresentation of d-loop NUMTs in the germline may be due to protein binding sites located in this region, which may interrupt mtDNA fragmentation and immigration to the nucleus. We propose an alternative mechanism which involves structural alteration of the chromosome. Under this model, the inserted mitochondrial d-loop insertion functions as a telomeric tloop, whereby it stabilizes a double-strand break and truncates the chromosome arm, resulting in aneuploidy. This mechanism involves transfer of the mtDNA displacement loop (d-loop) to the nuclear genome, where it could promote aneusomy by functioning as a telomeric "t-loop" structure, which typically caps the linear DNA molecule with a triple-stranded loop. The insertion of a mitochondrial d-loop could therefore directly interfere with the secondary structure of open chromatin and histone binding sites, leading to genome instability and/or dysregulation of gene expression. The second putative mechanism involves a mismatch between nucleotide composition between the mtDNA origin and nuclear DNA insertion site, which again draws from comparisons with work of Tsuji and coauthors [25]. Previous work has shown that non-homologous recombination and transposable element insertion can lead to genome instability by modifying local methylation patterns and altering molecular thermodynamics, which could lead to dysregulation of the cell cycle and aneuploidy. These two putative mechanisms are not mutually exclusive and we anticipate that novel mechanisms will be revealed through further investigation.

\section{Conclusions}

Our study reveals that numtogenesis plays an important role in the development of cancer and that NUMTs may serve as a biomarker for tumorigenesis. This study also identifies YME1L1 as the first NUMT suppressor gene in human and demonstrate that inactivation of YME1L1 induces migration of mtDNA to the nuclear genome. Exploration of mtDNA migration into the cancer genome should provide impetus for further studies to identify the mechanism(s) underlying numtogenesis. 


\section{Additional files}

Additional file 1: Table S1. NUMT abundance in all the colorectal tumor samples $(n=57)$ used in this study. (XLSX $14 \mathrm{~kb}$ )

Additional file 2: Figure S1. NUMT density in tumor and normal genomes (sorted by disease (T2) and fold change in NUMT abundance (T7)). Each peripheral node represents a TCGA sample whose blood-derived normal and tumor genomes were used in this study. From the outside to the inside, tracks are ordered from 1 to 7 (T1-T7). T1: Sample gender where red nodes represent female and blue nodes represent male. T2: Disease type information. Rectal adenocarcinoma (READ) is rendered as green bands and colon adenocarcinoma (COAD) as red bands. T3: Age at initial pathologic diagnosis ranging from 30 to 90 years. White and black filled bars represent white and black race of the individual, respectively. T4: Red columns represent NUMT proportion in tumor genomes and green columns represent blood-derived normal NUMT proportion. T5: Vital status of the patients-red for deceased individuals and green for alive status. T6: Stage of tumor represented in grey scale-stage I white, stage II grey, stage III dark grey, stage IV black. T7: Fold change in NUMT abundance. Samples with $<1$ fold are rendered as colored bands: 1-4-fold, blue; 4-8-fold, green; 8-12-fold, yellow; 12-20-fold, orange; >20-fold, red. (TIF $10991 \mathrm{~kb}$ )

Additional file 3: Figure S2. NUMT density in tumor and normal genomes (sorted by disease (T2) and pathologic tumor stage (T6)). Each peripheral node represents a TCGA sample whose blood-derived normal and tumor genomes were used in this study. From the outside to the inside, tracks are ordered from 1 to 7 (T1-T7). T1: Sample gender where red nodes represent female and blue nodes represent male. T2: Disease type information. Rectal adenocarcinoma $(R E A D)$ is rendered as green bands and colon adenocarcinoma (COAD) as red bands. T3: Age at initial pathologic diagnosis ranging from 30 to 90 years. White and black filled bars represent white and black race of the individual, respectively. T4: Red columns represent NUMT proportion in tumor genomes and green columns represent blood-derived normal NUMT proportion. T5: Vital status of the patients - red for deceased individuals and green for alive status. T6: Stage of tumor represented in grey scale-stage I white, stage II grey, stage III dark grey, stage IV black. T7: Fold change in NUMT abundance. Samples with $<1$ fold are rendered as colored bands: 1-4-fold, blue; 4-8-fold, green; 8-12-fold, yellow; 12-20-fold, orange; >20-fold, red. (TIF $10662 \mathrm{~kb}$ )

\section{Abbreviations}

BAM: Binary alignment; CGHub: Cancer Genomics Hub; COAD: Colon adenocarcinoma; CRC: colorectal cancer; mtDNA: Mitochondrial DNA; mtRNA: Mitochondrial RNA; NUMT: nuclear mtDNA sequence; QC: quality control; READ: Rectum adenocarcinoma; TCGA: The Cancer Genome Atlas

\section{Acknowledgements}

We thank Dr. Andreas Ivessa for generously providing us with PTY62 (Yme1-1) yeast strains, Dr. Thomas Fox for the yeast expression construct pPT31-yYme1, Dr. Thomas Langer for pYX113-hYme1L1, and Dr. Diana Stojanovski for pRS414yYme1 constructs.

\section{Funding}

This study was supported by grants from the Veterans Administration $1101 \mathrm{BX} 001716$ and a NCTN-LAPS Program Translational Research Award to KKS and T32HL072757 (PI: HKT) to MWS.

\section{Availability of data and materials}

TCGA data sets are publicly available to researchers upon individual institutional IRB approval and approval from dbgap.

\section{Authors' contributions}

KKS and HKT conceived the project and designed the experiments. VS, MWS $A S, V P M$, and BS performed the experiments and analyzed the data. PB analyzed YME1L1 mutations in tumors. VS, MWS, HKT, BS, and KKS wrote the manuscript. All authors read and approved the final manuscript.

\section{Authors' information}

MWS and BS contributed equally to this study. MWS's current affiliation is the Department of Biological and Environmental Sciences, School of Natural Sciences and Mathematics, University of West Alabama, Livingston, Alabama.

\section{Competing interests}

The authors declare that they have no competing interests.

\section{Consent for publication}

Not applicable.

\section{Ethics approval and consent to participate}

Datasets utilized in part of this research fall under dbGaP's "Protected/ Controlled Data Access System", which warrants a multi-step protocol involving the IRB team of UAB for gaining complete access to the requested research data. The data access process was initiated by Dr. Hemant Tiwari through his dbGaP eRA Commons account by submitting a research proposal description. Upon successful submission and approval to use identified TCGA Sequencing data, the Genomic Data Commons (GDC) data portal was used to access authorized sequencing datasets. GDC's data download and storage protocol was followed carefully to maintain integrity of the downloaded data and ensure a secure sandbox was staged to store and analyze the datasets. The research data part of dbGaP's study ID 'phs000178.v9.p8' (referenced and approved by TCGA as Project ID 4538) was downloaded and analyzed as part of our research effort. Furthermore, we de-identified the sample IDs by replacing the original Barcode-based TCGA ID convention with our internal numeric ID format. No individual consent was acquired as the study utilized the TCGA database.

\section{Publisher's Note}

Springer Nature remains neutral with regard to jurisdictional claims in published maps and institutional affiliations.

\section{Author details}

'Department of Biostatistics, School of Public Health, University of Alabama at Birmingham, Birmingham, Alabama 35294, USA. ${ }^{2}$ Department of Genetics, University of Alabama at Birmingham, Birmingham, Alabama 35294, USA. ${ }^{3}$ Departments of Genetics, Environmental Health, Center for Free Radical Biology, Center for Aging and UAB Comprehensive Cancer Center, University of Alabama at Birmingham, Birmingham, Alabama 35294, USA. ${ }^{4}$ Departments of Pathology, Environmental Health, Center for Free Radical Biology, Center for Aging and UAB Comprehensive Cancer Center, University of Alabama at Birmingham, Birmingham, Alabama 35294, USA. ${ }^{5}$ Birmingham Veterans Affairs Medical Center, Birmingham, Alabama 35294, USA. ${ }^{6}$ Department of Genetics, School of Medicine, University of Alabama at Birmingham, Kaul Genetics Building, Suite 620, 720 20th St. South, Birmingham, AL 35294, USA. ${ }^{\text {Present }}$ address: Department of Biological and Environmental Sciences, School of Natural Sciences and Mathematics, University of West Alabama, Livingston, Alabama, USA.

Received: 3 November 2016 Accepted: 9 March 2017

Published online: 29 March 2017

\section{References}

1. Hazkani-Covo E, Zeller RM, Martin W. Molecular poltergeists: mitochondrial DNA copies (numts) in sequenced nuclear genomes. PLoS Genet. 2010;6:e1000834.

2. Bakeeva LE, Skulachev VP, Sudarikova YV, Tsyplenkova VG. Mitochondria enter the nucleus (one further problem in chronic alcoholism). Biochemistry (Mosc). 2001;66:1335-41.

3. Bloom GD. A nucleus with cytoplasmic features. J Cell Biol. 1967;35:266-8.

4. Brandes D, Schofield BH, Anton E. Nuclear mitochondria? Science. 1965; 149:1373-4.

5. De Vos WH, Houben F, Kamps M, Malhas A, Verheyen F, Cox J, et al. Repetitive disruptions of the nuclear envelope invoke temporary loss of cellular compartmentalization in laminopathies. Hum Mol Genet. 2011;20: 4175-86.

6. Landerer E, Villegas J, Burzio VA, Oliveira L, Villota C, Lopez C, et al. Nuclear localization of the mitochondrial ncRNAs in normal and cancer cells. Cell Oncol (Dordr). 2011;34:297-305. 
7. Matsuyama M, Suzuki H. Seizing mechanism and fate of intranuclear mitochondria. Experientia. 1972;28:1347-8.

8. Sunba MS, Rahi AH, Morgan G. Tumours of the anterior uvea. II. Intranuclear cytoplasmic inclusions in malignant melanoma of the iris. Br J Ophthalmol. 1980;64:453-6.

9. Takemura G, Takatsu Y, Sakaguchi H, Fujiwara H. Intranuclear mitochondria in human myocardial cells. Pathol Res Pract. 1997;193:305-11.

10. Farrelly F, Butow RA. Rearranged mitochondrial genes in the yeast nuclear genome. Nature. 1983;301:296-301.

11. Zullo S, Sieu LC, Slightom JL, Hadler HI, Eisenstadt JM. Mitochondrial D-loop sequences are integrated in the rat nuclear genome. J Mol Biol. 1991;221: $1223-35$.

12. Dayama G, Emery SB, Kidd JM, Mills RE. The genomic landscape of polymorphic human nuclear mitochondrial insertions. Nucleic Acids Res. 2014:42:12640-9.

13. Hazkani-Covo E, Covo S. Numt-mediated double-strand break repair mitigates deletions during primate genome evolution. PLoS Genet. 2008;4 e1000237.

14. Wang D, Timmis JN. Cytoplasmic organelle DNA preferentially inserts into open chromatin. Genome Biol Evol. 2013;5:1060-4.

15. Woischnik M, Moraes $C T$. Pattern of organization of human mitochondrial pseudogenes in the nuclear genome. Genome Res. 2002;12:885-93.

16. Hallmann A, Milczarek R, Lipinski M, Kossowska E, Spodnik JH, Wozniak M, et al. Fast perinuclear clustering of mitochondria in oxidatively stressed human choriocarcinoma cells. Folia Morphol (Warsz). 2004;63:407-12.

17. Kim SJ, Syed GH, Siddiqui A. Hepatitis C virus induces the mitochondrial translocation of Parkin and subsequent mitophagy. PLoS Pathog. 2013;9:e1003285.

18. Villa AM, Doglia SM. Mitochondria in tumor cells studied by laser scanning confocal microscopy. J Biomed Opt. 2004;9:385-94

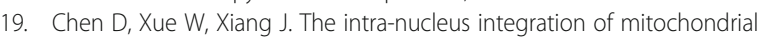
DNA (mtDNA)in cervical mucosa cells and its relation with c-myc expression. J Exp Clin Cancer Res. 2008;27:36.

20. Hadler HI, Devadas K, Mahalingam R. Selected nuclear LINE elements with mitochondrial-DNA-like inserts are more plentiful and mobile in tumor than in normal tissue of mouse and rat. J Cell Biochem. 1998:68:100-9.

21. Mourier T. Reverse transcription in genome evolution. Cytogenet Genome Res. 2005;1 10:56-62.

22. Mourier T, Hansen AJ, Willerslev E, Arctander P. The Human Genome Project reveals a continuous transfer of large mitochondrial fragments to the nucleus. Mol Biol Evol. 2001;18:1833-7.

23. Bensasson D, Feldman MW, Petrov DA. Rates of DNA duplication and mitochondrial DNA insertion in the human genome. J Mol Evol. 2003;57:343-54.

24. Lang M, Sazzini M, Calabrese FM, Simone D, Boattini A, Romeo G, et al. Polymorphic NumtS trace human population relationships. Hum Genet. 2012;131:757-71

25. Tsuji J, Frith MC, Tomii K, Horton P. Mammalian NUMT insertion is nonrandom. Nucleic Acids Res. 2012;40:9073-88

26. Zhang Z, Harrison PM, Liu Y, Gerstein M. Millions of years of evolution preserved: a comprehensive catalog of the processed pseudogenes in the human genome. Genome Res. 2003;13:2541-58.

27. Simone D, Calabrese FM, Lang M, Gasparre G, Attimonelli M. The reference human nuclear mitochondrial sequences compilation validated and implemented on the UCSC genome browser. BMC Genomics. 2011;12:517.

28. Amuthan G, Biswas G, Zhang SY, Klein-Szanto A, Vijayasarathy C, Avadhani NG. Mitochondria-to-nucleus stress signaling induces phenotypic changes, tumor progression and cell invasion. EMBO J. 2001;20:1910-20.

29. Delsite R, Kachhap S, Anbazhagan R, Gabrielson E, Singh KK. Nuclear genes involved in mitochondria-to-nucleus communication in breast cancer cells. Mol Cancer. 2002;1:6

30. Desler C, Munch-Petersen B, Stevnsner T, Matsui S, Kulawiec M, Singh KK, et al. Mitochondria as determinant of nucleotide pools and chromosomal stability. Mutat Res. 2007;625:112-24.

31. Donthamsetty S, Brahmbhatt M, Pannu V, Rida PC, Ramarathinam S, Ogden $A$, et al. Mitochondrial genome regulates mitotic fidelity by maintaining centrosomal homeostasis. Cell Cycle. 2014;13:2056-63.

32. Frezza $C$. The role of mitochondria in the oncogenic signal transduction. Int J Biochem Cell Biol. 2014;48:11-7.

33. Rasmussen AK, Chatterjee A, Rasmussen LJ, Singh KK. Mitochondriamediated nuclear mutator phenotype in Saccharomyces cerevisiae. Nucleic Acids Res. 2003;31:3909-17.
34. Singh KK, Kulawiec M, Still I, Desouki MM, Geradts J, Matsui S. Inter-genomic cross talk between mitochondria and the nucleus plays an important role in tumorigenesis. Gene. 2005;354:140-6.

35. Wallace DC. Mitochondria and cancer. Nat Rev Cancer. 2012:12:685-98.

36. Qu F, Liu X, Zhou F, Yang H, Bao G, He X, et al. Association between mitochondrial DNA content in leukocytes and colorectal cancer risk: a casecontrol analysis. Cancer. 2011;117:3148-55.

37. Thyagarajan B, Wang R, Barcelo H, Koh WP, Yuan JM. Mitochondrial copy number is associated with colorectal cancer risk. Cancer Epidemiol Biomarkers Prev. 2012;21:1574-81.

38. Webb E, Broderick P, Chandler I, Lubbe S, Penegar S, Tomlinson IP, et al Comprehensive analysis of common mitochondrial DNA variants and colorectal cancer risk. Br J Cancer. 2008:99:2088-93.

39. Skonieczna K, Malyarchuk BA, Grzybowski T. The landscape of mitochondrial DNA variation in human colorectal cancer on the background of phylogenetic knowledge. Biochim Biophys Acta. 2012;1825:153-9.

40. Theodoratou E, Din FV, Farrington SM, Cetnarskyj R, Barnetson RA, Porteous $M E$, et al. Association between common mtDNA variants and all-cause or colorectal cancer mortality. Carcinogenesis. 2010;31:296-301.

41. Bensasson D, Zhang D, Hartl DL, Hewitt GM. Mitochondrial pseudogenes: evolution's misplaced witnesses. Trends Ecol Evol. 2001;16:314-21.

42. Chen T, He J, Shen L, Fang H, Nie H, Jin T, et al. The mitochondrial DNA 4,977-bp deletion and its implication in copy number alteration in colorectal cancer. BMC Med Genet. 2011;12:8.

43. Lee HC, Yin PH, Lin JC, Wu CC, Chen CY, Wu CW, et al. Mitochondrial genome instability and mtDNA depletion in human cancers. Ann N Y Acad Sci. 2005;1042:109-22

44. Polyak K, Li Y, Zhu H, Lengauer C, Willson JK, Markowitz SD, et al. Somatic mutations of the mitochondrial genome in human colorectal tumours. Nat Genet. 1998:20:291-3.

45. Wilks C, Cline MS, Weiler E, Diehkans M, Craft B, Martin C, et al. The Cancer Genomics Hub (CGHub): overcoming cancer through the power of torrential data. Database (Oxford). 2014.

46. Chu HW, Rios C, Huang C, Wesolowska-Andersen A, Burchard EG, O'Connor $\mathrm{BP}$, et al. CRISPR-Cas9-mediated gene knockout in primary human airway epithelial cells reveals a proinflammatory role for MUC18. Gene Ther. 2015; 22:822-9.

47. West AP, Khoury-Hanold W, Staron M, Tal MC, Pineda CM, Lang SM, et al. Mitochondrial DNA stress primes the antiviral innate immune response. Nature. 2015;520:553-7.

48. Gietz RD, Schiestl RH, Willems AR, Woods RA. Studies on the transformation of intact yeast cells by the LiAc/SS-DNA/PEG procedure. Yeast. 1995;11:355-60.

49. Singh KK, Sigala B, Sikder HA, Schwimmer C. Inactivation of Saccharomyces cerevisiae OGG1 DNA repair gene leads to an increased frequency of mitochondrial mutants. Nucleic Acids Res. 2001:29:1381-8.

50. Soni R, Carmichael JP, Murray JA. Parameters affecting lithium acetatemediated transformation of Saccharomyces cerevisiae and development of a rapid and simplified procedure. Curr Genet. 1993;24:455-9.

51. Velarde MC. Mitochondrial and sex steroid hormone crosstalk during aging. Longev Healthspan. 2014;3:2

52. Bernardi G. Isochores and the evolutionary genomics of vertebrates. Gene. 2000;241:3-17

53. Saccone S, Federico C, Solovei I, Croquette MF, Della Valle G, Bernardi G Identification of the gene-richest bands in human prometaphase chromosomes. Chromosome Res. 1999:7:379-86.

54. Chen TL, Manuelidis L. SINEs and LINEs cluster in distinct DNA fragments of Giemsa band size. Chromosoma. 1989:98:309-16.

55. Thorsness PE, Fox TD. Escape of DNA from mitochondria to the nucleus in Saccharomyces cerevisiae. Nature. 1990;346:376-9.

56. Shah ZH, Hakkaart GA, Arku B, de Jong L, van der Spek H, Grivell LA, et al. The human homologue of the yeast mitochondrial AAA metalloprotease Yme1p complements a yeast yme1 disruptant. FEBS Lett. 2000:478:267-70.

57. Thorsness PE, Fox TD. Nuclear mutations in Saccharomyces cerevisiae that affect the escape of DNA from mitochondria to the nucleus. Genetics. 1993; 134:21-8.

58. Cheng $X$, Ivessa AS. The migration of mitochondrial DNA fragments to the nucleus affects the chronological aging process of Saccharomyces cerevisiae. Aging Cell. 2010;9:919-23.

59. Chen JM, Chuzhanova N, Stenson PD, Ferec C, Cooper DN. Meta-analysis of gross insertions causing human genetic disease: novel mutational mechanisms and the role of replication slippage. Hum Mutat. 2005;25:207-21. 
60. Borensztajn K, Chafa O, Alhenc-Gelas M, Salha S, Reghis A, Fischer AM, et al. Characterization of two novel splice site mutations in human factor VII gene causing severe plasma factor VII deficiency and bleeding diathesis. $\mathrm{Br}$ J Haematol. 2002;117:168-71.

61. Goldin E, Stahl S, Cooney AM, Kaneski CR, Gupta S, Brady RO, et al. Transfer of a mitochondrial DNA fragment to MCOLN1 causes an inherited case of mucolipidosis IV. Hum Mutat. 2004;24:460-5.

62. Ahmed ZM, Smith TN, Riazuddin S, Makishima T, Ghosh M, Bokhari S, et al. Nonsyndromic recessive deafness DFNB18 and Usher syndrome type IC are allelic mutations of USHIC. Hum Genet. 2002;110:527-31.

63. Turner C, Killoran C, Thomas NS, Rosenberg M, Chuzhanova NA, Johnston J, et al. Human genetic disease caused by de novo mitochondrial-nuclear DNA transfer. Hum Genet. 2003;112:303-9.

64. Miettinen TP, Bjorklund M. Cellular Allometry of mitochondrial functionality establishes the optimal cell size. Dev Cell. 2016:39:370-82.

65. Shay JW, Baba T, Zhan QM, Kamimura N, Cuthbert JA. HeLaTG cells have mitochondrial DNA inserted into the c-myc oncogene. Oncogene. 1991;6:1869-74.

66. Hu Y, Qian G, Mao B, Xiao T, Li Y, Cao S. Malignant transformation of mouse embryonic fibroblast induced by mitochondrial DNA fragments. Zhonghua Bing Li Xue Za Zhi. 2000;29:39-42.

67. de Araujo LF, Fonseca AS, Muys BR, Placa JR, Bueno RB, Lorenzi JC, et al. Mitochondrial genome instability in colorectal adenoma and adenocarcinoma. Tumour Biol. 2015;36:8869-79.

68. Hsu CC, Tseng LM, Lee HC. Role of mitochondrial dysfunction in cancer progression. Exp Biol Med (Maywood). 2016;241:1281-95.

69. Kulawiec M, Safina A, Desouki MM, Still I, Matsui S, Bakin A, et al. Tumorigenic transformation of human breast epithelial cells induced by mitochondrial DNA depletion. Cancer Biol Ther. 2008;7:1732-43.

70. Modica-Napolitano JS, Kulawiec M, Singh KK. Mitochondria and human cancer. Curr Mol Med. 2007;7:121-31.

71. Owens KM, Kulawiec M, Desouki MM, Vanniarajan A, Singh KK. Impaired OXPHOS complex III in breast cancer. PLoS ONE. 2011;6:e23846.

72. Singh KK. Mitochondrial dysfunction is a common phenotype in aging and cancer. Ann N Y Acad Sci. 2004;1019:260-4.

73. Abdel-Rahman WM. Genomic instability and carcinogenesis: an update. Curr Genomics. 2008;9:535-41.

74. Giam M, Rancati G. Aneuploidy and chromosomal instability in cancer: a jackpot to chaos. Cell Div. 2015:10:3.

75. Modica-Napolitano JS, Singh KK. Mitochondrial dysfunction in cancer. Mitochondrion. 2004:4:755-62.

76. Hadler HI, Daniel BG, Pratt RD. The induction of ATP energized mitochondrial volume changes by carcinogenic N-hydroxy-N-acetylaminofluorenes when combined with showdomycin. A unitary hypothesis for carcinogenesis. J Antibiot (Tokyo). 1971;24:405-17.

77. Akouchekian M, Houshmand M, Akbari MH, Kamalidehghan B, Dehghan M. Analysis of mitochondrial ND1 gene in human colorectal cancer. J Res Med Sci. 2011;16:50-5.

78. Wang CY, Li H, Hao XD, Liu J, Wang JX, Wang WZ, et al. Uncovering the profile of somatic mtDNA mutations in Chinese colorectal cancer patients. PLOS ONE. 2011;6:e21613.

79. Francis BR, Thorsness PE. Hsp90 and mitochondrial proteases Yme1 and Yta10/12 participate in ATP synthase assembly in Saccharomyces cerevisiae. Mitochondrion. 2011:11:587-600.

80. Wang $\mathrm{K}$, Jin M, Liu X, Klionsky DJ. Proteolytic processing of Atg32 by the mitochondrial i-AAA protease Yme1 regulates mitophagy. Autophagy. 2013;9: 1828-36.

81. Deffieu M, Bhatia-Kissova I, Salin B, Klionsky DJ, Pinson B, Manon S, et al. Increased levels of reduced cytochrome $b$ and mitophagy components are required to trigger nonspecific autophagy following induced mitochondrial dysfunction. J Cell Sci. 2013;126:415-26.

82. Oka T, Hikoso S, Yamaguchi O, Taneike M, Takeda T, Tamai T, et al. Mitochondrial DNA that escapes from autophagy causes inflammation and heart failure. Nature. 2012;485:251-5.

83. Jensen $H$, Engedal $H$, Saetersdal TS. Ultrastructure of mitochondria-containining nuclei in human myocardial cells. Virchows Arch B Cell Pathol. 1976;21:1-12.

84. Thorsness PE, Weber ER. Escape and migration of nucleic acids between chloroplasts, mitochondria, and the nucleus. Int Rev Cytol. 1996;165:207-34.

85. Guttinger $\mathrm{S}$, Laurell $\mathrm{E}$, Kutay U. Orchestrating nuclear envelope disassembly and reassembly during mitosis. Nat Rev Mol Cell Biol. 2009;10:178-91.

86. Zink D, Fischer AH, Nickerson JA. Nuclear structure in cancer cells. Nat Rev Cancer. 2004;4:677-87.
87. Vargas JD, Hatch EM, Anderson DJ, Hetzer MW. Transient nuclear envelope rupturing during interphase in human cancer cells. Nucleus. 2012;3:88-100.

88. de Las Heras JI, Batrakou DG, Schirmer EC. Cancer biology and the nuclear envelope: a convoluted relationship. Semin Cancer Biol. 2013:23:125-37.

\section{Submit your next manuscript to BioMed Central and we will help you at every step:}

- We accept pre-submission inquiries

- Our selector tool helps you to find the most relevant journal

- We provide round the clock customer support

- Convenient online submission

- Thorough peer review

- Inclusion in PubMed and all major indexing services

- Maximum visibility for your research

Submit your manuscript at www.biomedcentral.com/submit
Biomed Central 